Planetary Systems in the Universe - Observation, Formation and Evolution

Proceedings IAU Symposium No. 202, (c)2004 IAU

Alan Penny, Pawel Artymowicz, Anne-Marie Lagrange, 8 Sara Russell, eds.

\title{
Masers as probes of proto-planetary discs
}

\author{
A.M.S. Richards, R.J. Cohen, M. Crocker \\ JBO, University of Manchester, Cheshire, SK11 9DL. UK
}

E.E. Lekht

INAOEP, Apartado Postal 51 y 216, 72000, Puebla, Pue. Mexico

V. Samodourov, I.I. Berulis

LPI, 4/32 Profsoyuznaya st., Moscow, 117810, Russia

A. Lobanov

MPIfRA, Auf dem Högel 69, D-53121 Bonn, Germany

L. Moscadelli

Stazione Astronomica, Strada 54, 09012 Capoterra (CA), Italy

\begin{abstract}
Water maser emission from star forming regions has been monitored for several decades using the Puschino radio telescope, showing radial velocity variations consistent with material in Keplerian orbit around protostars. MERLIN and the EVN are now being used to image the $22 \mathrm{GHz}$ emission on au scales and measure proper motions. This will distinguish discs from outflows, and provide an estimate of the central mass and possibly orbiting condensations.
\end{abstract}

\section{Introduction}

Masers are the brightest spectral lines found in star-forming regions. Recently, $\mathrm{CH}_{3} \mathrm{OH}$ masers have shown clear evidence for Keplerian rotation in protostellar disks (Norris et al. 1998; Minier, Booth, \& Conway 2000) A\&A). $\mathrm{H}_{2} \mathrm{O}$ masers have usually been associated with shocked outflows but protoplanetary discs may also produce suitable conditions. Since 1980 a joint LPI-SAI group has monitored about fifty $22-\mathrm{GHz} \mathrm{H}_{2} \mathrm{O}$ maser sources using the Puschino radio telescope e.g. Lekht et al. (1993). A number of objects show a triplet of persistent spectral features with small velocity drifts which are sometimes correlated with brightness changes. Four possible models include accelerating outflows or Keplerian rotation. Intriguingly, if orbital motion is being observed, irregularities in Keplerian behaviour suggest discrete clumps. Can $\mathrm{H}_{2} \mathrm{O}$ masers be used to trace matter as it condenses in a protostellar disk? 
Masers as probes of proto-planetary discs

S140 $22 \mathrm{GHz}$ Scalar averaged cross-power spectrum

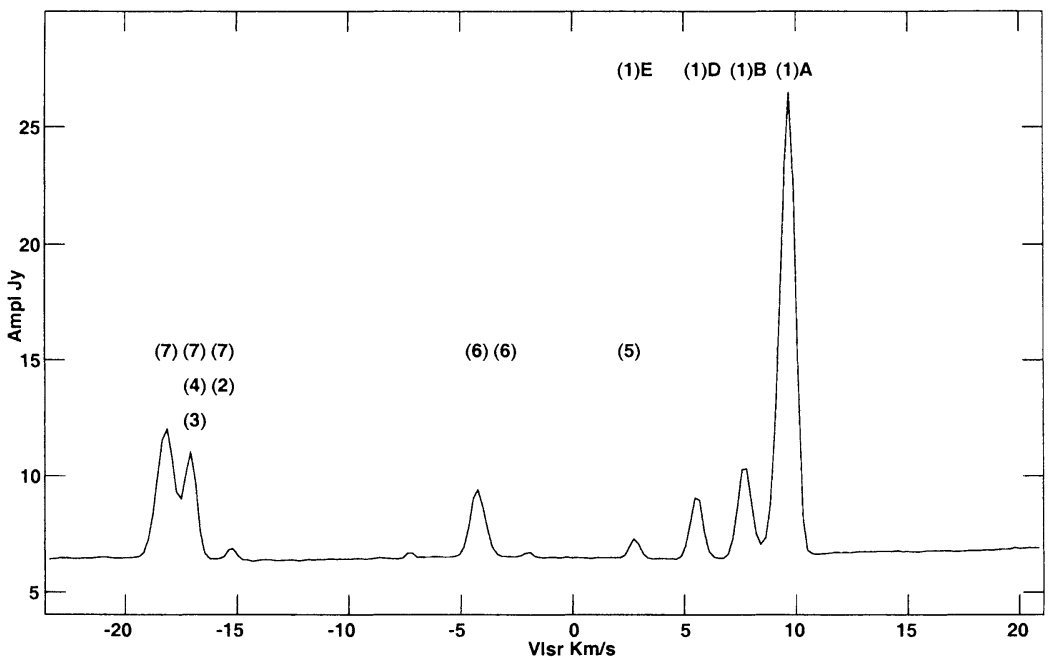

Figure 1. Velocity profile of S1240 observed with MERLIN in 1998.

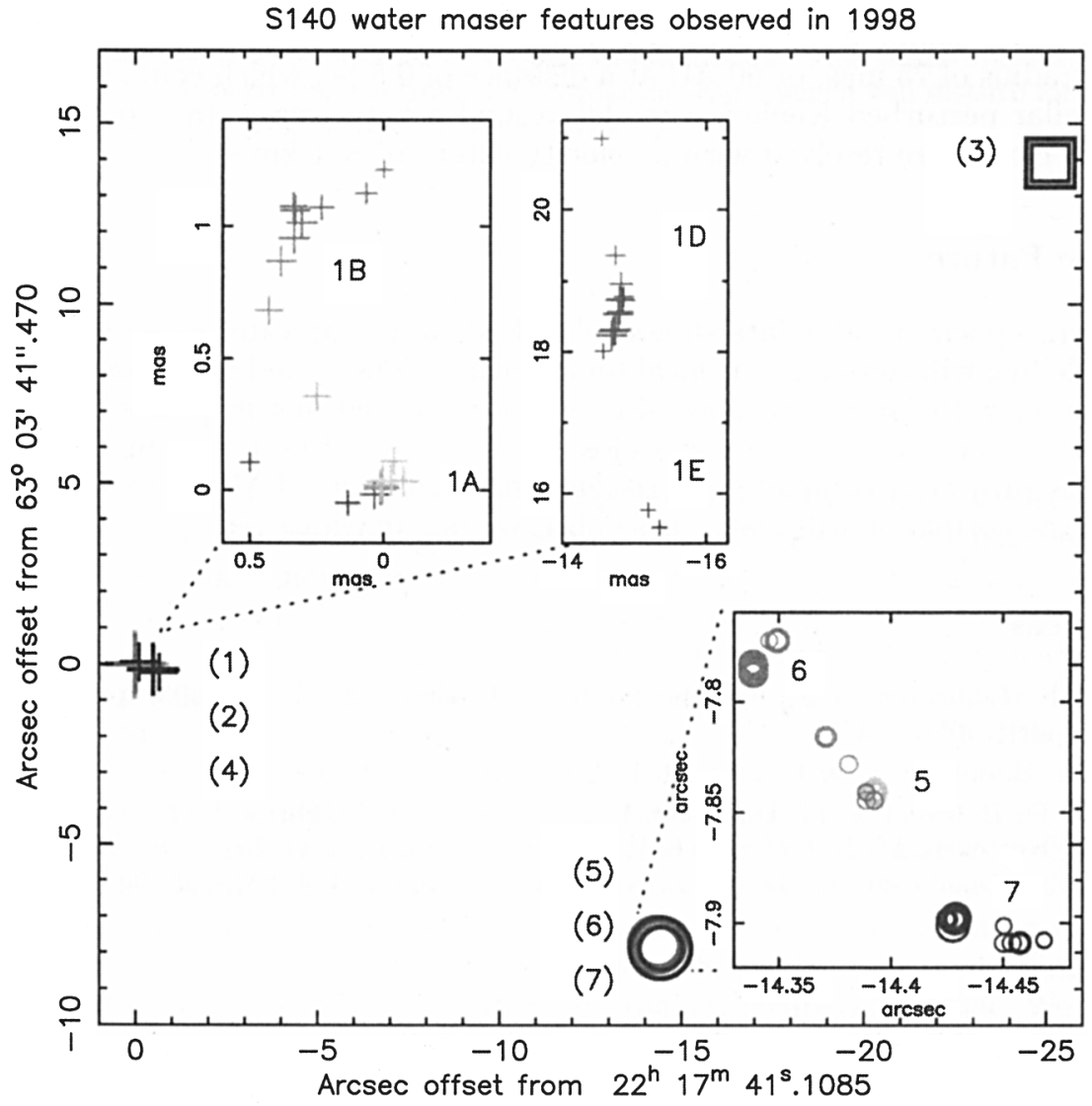

Figure 2. Location of $\mathrm{H}_{2} \mathrm{O}$ masers, labeled as in Fig. 1 


\section{Observations}

MERLIN ${ }^{1}$ has milli-arcsec (mas) resolution at $22 \mathrm{GHz}$ in $0.1 \mathrm{~km} \mathrm{~s}^{-1}$ velocity channels. An absolute position accuracy of 10 mas can be achieved. A series of observations of five nearby sources started in 1998 to measure $\mathrm{H}_{2} \mathrm{O}$ maser clump size and distribution and proper motions. This will distinguish rotation from outflow in a few years. Other sources (at greater distances, requiring higher resolution) are being observed by the European VLBI Network.

\section{Preliminary Results}

The first epoch of MERLIN observations showed that some velocity peaks are blends of emission from separate maser sources up to $50^{\prime \prime}$ apart, but each spatial region is distinct and covers less than an arcsec with a complex but non-random velocity structure. For example S140 has a maximum 22-GHz spectral peak separation of $\sim 25 \mathrm{~km} \mathrm{~s}^{-1}$ (Fig. 1). Lekht et al. (1993) fitted models to the spectral variations which suggested the masers emanate from fragments $\geq 1$ au in diameter, orbiting at $25-80 \mathrm{AU}$ from the central star, mass $5 \mathrm{M}_{\odot}$. Imaging (Fig. 2) shows several separate regions which could be associated with distinct protostars; for example the region 5-6-7 has a $20 \mathrm{~km} \mathrm{~s}^{-1}$ velocity range and an angular radius of 75 mas, or $60 \mathrm{AU}$ at a distance of $0.8 \mathrm{pc}$, which could be fitted by a similar perturbed Keplerian model around a lower mass star. Individual AU-scale clouds are resolved with a velocity extent of $\sim 1 \mathrm{~km} \mathrm{~s}^{-1}$.

\section{The Future}

This single epoch of radio data shows sub-AU structure of water vapour clouds, and modelling will constrain the local temperature, density and other conditions. Comparison with later data may also reveal condensations in protoplanetary discs, but in any case gas dynamics close to the star will be better understood when this project is complete. Increasing the sensitivity of MERLIN and upgrading the correlator will make these observations much easier.

\section{References}

Lekht, E. E., Likhachev, S. F., Sorochenko, R. L., \& Strel'ntskii, V. S. 1993, Astronomy Reports, 37(4), 367L

Minier, V., Booth, R. S., \& Conway, J. E. 2000, A\&A, in press

Norris, R. P., Byleveld, S. E., Diamond, P. J., Ellingsen, S. P., Ferris, R. H., Gough, R. G., Kesteven, M. J., McCulloch, P. M., Phillips, C. J., Reynolds, J. E., Tzioumis, A. K., Takahashi, Y., Troup, E. R., \& Wellington, K. J. 1998, ApJ, 508, 275

\footnotetext{
${ }^{1}$ MERLIN is the UK national radio astronomy facility operated by the University of Manchester at Jodrell Bank Observatory on behalf of PPARC
} 